\title{
Description of a high-risk region as a control object
}

\author{
A.B. Sadigov ${ }^{a *}$, I.I. Mustafayev ${ }^{\text {b }}$, G.N. Hajimatov ${ }^{\text {b }}$ E.K. Gafarov ${ }^{b}$ \\ ${ }^{a}$ Institute of Control Systems of Azerbaijan National Academy of Sciences, Baku, Azerbaijan \\ ${ }^{b}$ Azerbaijan University of Architecture and Construction, Baku, Azerbaijan
}

\begin{tabular}{|c|c|}
\hline $\begin{array}{ll}\text { A R T I C L E I N F O } & \text { I }\end{array}$ & A B S T RA C T \\
\hline $\begin{array}{l}\text { Article history: } \\
\text { Received 15.04.2021 } \\
\text { Received in revised form } 27.04 .2021 \\
\text { Accepted } 12.05 .2021 \\
\text { Available online } 29.12 .2021 \\
\text { Keywords: } \\
\text { Mathematical modeling } \\
\text { High-risk region } \\
\text { Emergencies } \\
\text { Military-technogenic factors } \\
\text { Regional protection management }\end{array}$ & $\begin{array}{l}\text { The functioning of a high-risk region in emergency situations } \\
\text { (ES) is considered as a process of serial-parallel changes in the } \\
\text { states of regional components. The general regional protection } \\
\text { management problem is divided into three main tasks, which are } \\
\text { formulated for the threat, crisis and post-crisis periods of the } \\
\text { development of an emergency. }\end{array}$ \\
\hline
\end{tabular}

\section{Introduction}

Domestic and foreign experience shows that in order to improve the management of protection actions in emergency situations, it is advisable to use an automated system in each highrisk region to provide prompt and coordinated solution of problems in a hierarchical management structure [1]. The specifics of the construction and functioning of such a system is associated with territorial distribution, heterogeneity, multi-connectivity, dynamism and initial uncertainty of the factors of destruction and protection in an emergency. The consistent refinement and expansion of formalized representations of the automation object is provided by an iterative system design procedure carried out on the basis of the professional experience of experts, specific functional requests of users, methodological and technical input of developers.

\section{Problem statement}

The functioning of a region in emergency situations (ES) is considered as a process of serialparallel changes in the states of regional components [1]. Therefore, it can be represented as a formal system:

$$
\Sigma=(T, X, U, Y, H, G),
$$

where $T$ is the set of time components; $X$ is the set of states of the system; $U$ is the set of admissible input actions; $Y$ is the set of output quantities; $H: T \times X \times U \rightarrow X$ is the operator of system

\footnotetext{
${ }^{*}$ Corresponding author. gadjimetov@bk.ru (G.N. Hajimatov), emilqafarov@inbox.ru (E.K. Gafarov).

E-mail addresses: aminaga.sadigov@gmail.com (A.B. Sadigov), imustafayev@mail.ru (I.I. Mustafayev), 
transitions; $G: T \times X \times U \rightarrow Y$ is the operator of system outputs.

The set of instants of time $T$, at which the functioning of the regional system is considered, is specified by an ordered subset of the set of real numbers. At any instant of time $t \in T$, the state of the system $X_{t} \in X$ is represented by the state vector of objects of destruction and protection:

$$
X_{t}=\left(X_{1 t}, \ldots, X_{\alpha t}, \ldots, X_{n t}\right)
$$

The set of alternative states of each object $X_{\alpha t}$ in the time interval $T$ will be denoted by $X_{\alpha t}$. The state space (or phase space) of the system is a direct product:

$$
X_{T}=X_{1 T} \times \ldots \times X_{\alpha t} \times \ldots \times X_{n t}
$$

The set of admissible input actions $U$ is naturally broken down into two non-overlapping subsets: protective actions $M$ and destructive actions $W$. At the same time, admissible protective actions are possible states of engaged resources $Z$, and admissible destructive actions are possible states of identified sources $V$.

Protective actions can be carried out at any time instant $t \in T$ by the resources $Z_{t} \in Z$, which form the vector:

$$
Z_{t}=\left(Z_{1 t}, \ldots, Z_{\beta t}, \ldots, Z_{m t}\right)
$$

If we denote by $Z_{\beta t}$ the set of alternative states of each resource $Z_{\beta t}$ on the interval $T$, the direct product:

$$
Z_{T}=Z_{1 T} \times \ldots \times Z_{\beta t} \times \ldots \times Z_{m t}
$$

is the space of protective actions.

Destructive actions can also manifest themselves at any time instant $t \in T$ from the sources $V_{t} \in V$, which form the vector:

$$
V_{t}=\left(V_{1 t}, \ldots, V_{\gamma t}, \ldots, V_{q t}\right)
$$

The space of destructive actions is represented by the direct product:

$$
V_{T}=V_{1 T} \times \ldots \times V_{\gamma T} \times \ldots \times V_{q t},
$$

where $V_{\gamma T}$ is the set of alternative states of each source $V_{\gamma T}$ on the intervalT.

The output quantities $Y_{t} \in Y$ that characterize the functioning of the system at any time instant $t \in T$ form the vector of characteristics:

$$
Y_{t}=\left(Y_{1 t}, \ldots, Y_{j t}, \ldots, Y_{p t}\right)
$$

If $Y_{j t}$ is the set of different values of each characteristic on the interval $T$, then the direct product

$$
Y_{T}=Y_{1 T} \times \ldots \times Y_{j t} \times \ldots \times Y_{p t}
$$

is called the space of output quantities.

The operator of transitions $M$ determines the trajectory of the systems in the phase space $X_{T}$ depending on the input actions $M$ and $W$, i.e., $H=M \times W$. These actions are oppositely directed. Therefore, two domains are distinguished in the space $X_{T}$ : desirable states $X_{T}^{\xi}$ and undesirable states $X_{T}^{\omega}$. The domain $X_{T}^{\xi}$ is formed when the system moves in the space of protective actions:

$$
M: X_{T} \times Z_{T} \rightarrow X_{T}^{\xi}
$$

and the domain $X_{T}^{\omega}$ is formed when the system moves in the space of destructive actions:

$$
W: X_{T} \times V_{T} \rightarrow X_{T}^{\omega}
$$




\section{Solution}

The purposeful functioning of the system occurs when protective actions are carried out that keep or transfer the system to the domain of desirable states, thereby counteracting its finding or transition to the domain of undesirable states. Effective management of these processes requires anticipating undesirable transitions and proactively take adequate protective actions. It follows that the operator $M$, which controls the transitions of the system to the desirable states $X_{T}^{\xi}$ on the interval $T$, should be built on the basis of anticipating its possible transitions in the same time interval to undesirable states desirable from the expected sources $V_{T}$ :

$$
M: X_{T}^{\omega} \times Z_{T} \times V_{T} \rightarrow X_{T}^{\xi}
$$

The operator $M$ in representation (2) is implemented as a logically interconnected system of protective actions carried out by the resources $Z_{T}$. The management of these activities (resources) should form such a trajectory of the system movement that ensures the achievement of its target state $X_{T}^{\xi}$ on the bounded interval $T$.

The effectiveness of regional protection (RP) management characterized by operator $M$ depends on the ability to proactively counteract or compensate for the expected destructive actions from the sources $V_{T}$ by rational choice or allocation of protective action resources for objects of possible destruction $X_{T}^{\omega}$ on the forecast interval $T$ [2]. The general criterion for the effectiveness of regional protection (RP) management is the assessment of the degree of achievement by the system of the target state $X_{T}^{\xi}$ within the bounded interval $T$. This assessment is made on the basis of the output quantities $Y_{-} T$ characterizing the functioning of the system over the considered time interval. Considering that in representation (1), the output operator $G$ and the transition operator $H$ have the same domain of definition, the output quantities can be expressed in terms of states of the objects $X_{T}$, the resources $Z_{T}$, and the sources $V_{T}$. Accordingly, in the space of output quantities $Y_{T}$, we can distinguish the domain of characteristics of the desirable functioning of the system $Y_{T}^{\xi}=$ $\left(Z_{T}, X_{T}^{\xi}\right)$ and the domain of characteristics of its undesirable functioning $Y_{T}^{\omega}=\left(V_{T}, X_{T}^{\omega}\right)$. The indicated output quantities (characteristics) are considered at each time instant $t \in T$ as coordinates of the corresponding vectors $X_{t}, Z_{t}$ and $V_{t}$. Practical measurement of these characteristics to assess the effectiveness of RP management is possible using the indicators considered above when constructing an event-driven model of emergencies development. Different values of these indicators (quantitative or qualitative) characterize different states of regional components, and the general criterion for the effectiveness of RP management is the supplying of destruction and protection with protective action resources required for countering an unfavorable environment.

Since the specified indicators and their values significantly depend on the period of development of ES, we break down the set of time instants $T=\left[t_{0}, t_{N}\right]$ into the following subsets:

$\left[t_{1}, t_{u}\right] \in T_{u}$ - threat period,

$\left[t_{2}, t_{k}\right] \in T_{k}-$ crisis period,

$\left[t_{3}, t_{p}\right] \in T_{p}$ - post-crisis period,

where $T_{u} \cup T_{k} \cup T_{p} \in T ; t_{0} \leq t_{1}<t_{u} \leq t_{2}<t_{k} \leq t_{3}<t_{p} \leq t_{N}$.

Using the introduced notions and designations, we can represent indicators of the states of regional components as the following vectors:

a) indicators of desirable states of

- the resources $Z_{T}=\left(Z_{T_{u}}^{\Psi}, Z_{T_{k}}^{\sigma}, Z_{T_{p}}^{\theta}\right)$, 
where $Z_{T_{u}}^{\Psi}=U_{\beta} Z_{\beta T_{u}}^{\Psi} ; Z_{T_{k}}^{\sigma}=U_{\beta} Z_{\beta T_{k}}^{\sigma} ; Z_{T_{p}}^{\theta}=U_{\beta} Z_{\beta T_{p}}^{\theta}$;

- the objects $X_{T}^{\xi}=\left(X_{T_{u}}^{\Psi}, X_{T_{k}}^{\sigma}, X_{T_{p}}^{\theta}\right)$,

where $X_{T_{u}}^{\Psi}=\cup_{\alpha} \bigcup_{v} X_{\alpha T_{u}}^{\Psi v} ; X_{T_{k}}^{\sigma}=\bigcup_{\alpha} \bigcup_{v} X_{\alpha T_{k}}^{\sigma v} ; X_{T_{p}}^{\theta}=\bigcup_{\alpha} \bigcup_{v} X_{\alpha T_{p}}^{\theta v}$;

б) indicators of undesirable states of

- the sources $V_{T}=\left(V_{T_{u}}^{\rho}, V_{T_{k}}^{\pi}, V_{T_{p}}^{\delta}\right)$,

where $V_{T_{u}}^{\rho}=U_{\gamma} V_{\gamma T_{u}}^{\rho} ; V_{T_{k}}^{\pi}=U_{\gamma} V_{\gamma T_{k}}^{\pi} ; V_{T_{p}}^{\delta}=U_{\gamma} V_{\gamma T_{p}}^{\delta}$,

- the objects $X_{T}^{\omega}=\left(X_{T_{u}}^{\rho}, X_{T_{k}}^{\pi}, X_{T_{\rho}}^{\delta}\right)$,

where $X_{T_{u}}^{\rho}=\bigcup_{\alpha} \bigcup_{\mathfrak{x}} X_{\alpha T_{u}}^{\rho \mathfrak{x}} ; X_{T_{k}}^{\pi}=\bigcup_{\alpha} \bigcup_{\mathfrak{x}} X_{\alpha T_{k}}^{\pi \mathfrak{r}} ; X_{T_{\rho}}^{\delta}=\bigcup_{\alpha} \bigcup_{\mathfrak{x}} X_{\alpha T_{\rho}}^{\delta \mathfrak{r}}$.

An increase in the efficiency of RP management is manifested in a decrease in the values of indicators of undesirable states due to an increase in the values of indicators of desirable states. In accordance with the event-driven model of ES development [3], the overall target indicator $\max X_{T}^{\xi}$ is achieved consistently through the implementation of intermediate target indicators of the states of regional components in the threat, crisis and post-crisis periods.

The above allows us to break down the general problem of RP management according to the selected periods of ES development into three main tasks:

1) in the threat period - for a specified initial state of the objects $X_{\alpha t_{0}}$, the preventiveness of the resources $Z_{\beta t_{1}}^{\Psi}$ and the threat for the sources $V_{\gamma t_{1}}^{\rho}$, to determine on the bounded time interval $\left[t_{1}, t_{u}\right] \in T_{u}$ a vector of resources $Z_{\beta\left[t_{1}, t_{u}\right]}^{\Psi}$ such that provides maximum resistance for the objects $X_{\alpha\left[t_{1}, t_{u}\right]}^{\rho}$ located in the risk zones $V_{\gamma\left[t_{1}, t_{u}\right]}^{\rho}$ :

$$
\begin{aligned}
\max \left\{X_{\alpha\left[t_{1}, t_{u}\right]}^{\Psi}=\right. & \left.M^{\Psi}\left(X_{\alpha t_{0}}, X_{\alpha\left[t_{1}, t_{u}\right]}^{\rho}, Z_{\beta\left[t_{1}, t_{u}\right]}^{\Psi}, V_{\gamma\left[t_{1}, t_{u}\right]}^{\rho}\right)\right|_{Z_{\beta\left[t_{1}, t_{u}\right]}^{\Psi} \in Z_{T_{u}}^{\Psi}} \\
& X_{\alpha\left[t_{1}, t_{u}\right]}^{\rho}=W^{\rho}\left(X_{\alpha T_{0}}, V_{\gamma\left[t_{1}, t_{u}\right]}^{\rho}\right) ; \\
& \left.X_{\alpha\left[t_{1}, t_{u}\right]}^{\rho} \in X_{T_{u}}^{\rho} ; \quad V_{\gamma\left[t_{1}, t_{u}\right]}^{\rho} \in V_{T_{u}}^{\rho}\right\}
\end{aligned}
$$

2) in the crisis period - for a specified initial resistance of the objects $X_{\alpha t_{u}}^{\Psi}$, rescuability of the resources $Z_{\beta t_{2}}^{\sigma}$ and criticality of the sources $V_{\gamma t_{2}}^{\pi}$, to determine on the bounded time interval $\left[t_{2}, t_{k}\right] \in T_{k}$ a vector of resources $Z_{\beta\left[t_{2}, t_{k}\right]}^{\sigma}$ such that provides maximum survival for the objects $X_{\alpha\left[t_{2}, t_{k}\right]}^{\pi}$ in the destruction zones $V_{\gamma\left[t_{2}, t_{k}\right]}^{\pi}$ :

$$
\begin{aligned}
\max \left\{X_{\alpha\left[t_{2}, t_{k}\right]}^{\sigma}=\right. & \left.M^{\sigma}\left(X_{\alpha t_{u}}^{\Psi}, X_{\alpha\left[t_{2}, t_{k}\right]}^{\pi}, Z_{\beta\left[t_{2}, t_{k}\right]}^{\sigma}, V_{\gamma\left[t_{2}, t_{k}\right]}^{\pi}\right)\right|_{Z_{\beta\left[t_{2}, t_{k}\right]}^{\sigma} \in Z_{T_{k}}^{\sigma}} \\
& X_{\alpha\left[t_{2}, t_{k}\right]}^{\pi}=W^{\pi}\left(X_{\alpha t_{u}}^{\Psi}, V_{\gamma\left[t_{2}, t_{k}\right]}^{\pi}\right) ; \\
& \left.X_{\alpha\left[t_{2}, t_{k}\right]}^{\pi} \in X_{T_{k}}^{\pi} ; \quad V_{\gamma\left[t_{2}, t_{k}\right]}^{\pi} \in V_{T_{k}}^{\pi}\right\}
\end{aligned}
$$

3 ) in the post-crisis period - for a specified initial survivability of the objects $X_{\alpha t_{k}}^{\sigma}$, restorability of the resources $Z_{\beta t_{3}}^{\theta}$ and post-criticality of the sources $V_{\gamma t_{3}}^{\delta}$, to determine on the bounded time interval $\left[t_{3}, t_{p}\right] \in T_{\rho}$ a vector of resources $Z_{\beta\left[t_{3}, t_{p}\right]}^{\theta}$ such that provides maximum rehabilitation for the objects $X_{\alpha\left[t_{3}, t_{p}\right]}^{\delta}$ in the aftermath zones $V_{\gamma c\left[t_{3}, t_{p}\right]}^{\delta}$ : 


$$
\begin{aligned}
\max \left\{X_{\alpha\left[t_{3}, t_{p}\right]}^{\theta}=\right. & \left.M^{\theta}\left(X_{\alpha t_{k}}^{\sigma}, X_{\alpha\left[t_{3}, t_{p}\right]}^{\delta}, Z_{\beta\left[t_{3}, t_{p}\right]}^{\theta}, V_{\gamma\left[t_{3}, t_{p}\right]}^{\delta}\right)\right|_{Z_{\beta\left[t_{3}, t_{p}\right]}^{\theta} \in Z_{T_{p}}^{\theta}} \\
& X_{\alpha\left[t_{3}, t_{p}\right]}^{\delta}=W^{\delta}\left(X_{\alpha t_{k}}^{\sigma}, V_{\rho\left[t_{3}, t_{p}\right]}^{\delta}\right) \\
& \left.X_{\alpha\left[t_{3}, t_{p}\right]}^{\delta} \in X_{T_{\rho}}^{\delta} ; \quad V_{\gamma\left[t_{3}, t_{p}\right]}^{\delta} \in V_{T_{\rho}}^{\delta}\right\}
\end{aligned}
$$

\section{Conclusion}

In real-life conditions, a direct and accurate solution of the formulated problems is significantly hampered by the following circumstances:

- a rapidly changing situation requires a prompt reallocation (maneuvering) of limited forces and means, in each period of the development of the emergency;

- different departmental affiliation of specialized units leads to the need for continuous vertical and horizontal coordination of numerous services and agencies that manage regional protection in the course of the development of the emergency;

- the lack of up-to-date, reliable and complete information about the current situation complicates the preparation of prompt and well-grounded decisions on adequate counteraction against an unfavorable environment.

In practice, an approximate solution of these problems is carried out by breaking them down into relatively independent and simpler subtasks in the regional protection management system [4].

\section{References}

[1] А.Б. Садыгов, Модели и технологии решения задач управления в чрезвычайных ситуациях, Баку, “Элм”, (2017) 356 p. https://www.researchgate.net/profile/Aminaga_Sadigov [In Russian: A.B. Sadigov, Models and Technologies for Solving Control Problems in Emergency Situations, Baku, Elm].

[2] A.B. Sadigov, R.M. Zeynalov, Optimal control in the problems of calculating the benefit/cost ratio in emergency response, 40 No.1 (2020) p.47-56. https://icp.az/2020/1-06.pdf

[3] A.B. Sadigov, R.M. Zeynalov, Mathematical modeling of environmental processes at military facilities, Informatics and Control Problems. 40 No.2 (2020) p.31-37. https://icp.az/2020/2-04.pdf

[4] A.B. Sadigov, Methods of risks assessment of life activity in environment, 1st International Turkish World Engineering and Science Congress in Antalya, December 7-10, 2017, Turk. http://teskongre.org/web2/ing/w/2017/ 